\title{
A rare cause for gestational trophoblastic neoplasia: Placental site trophoblastic tumor
}

\author{
Volkan Ulker $^{1}$, Alpaslan Akyol ${ }^{2}$, Ali Gedikbasi ${ }^{2 *}$, Ceyhun Numanoglu ${ }^{1}$, Ergul Demircivi ${ }^{2}$, \\ Huseyin Sitki Tuzlali ${ }^{3}$ \\ ${ }^{1}$ Kanuni Sultan Suleyman Teaching and Research Hospital, Department of Obstetrics and Gynecology, Oncology Unit, Istanbul, \\ Turkey \\ ${ }^{2}$ Kanuni Sultan Suleyman Teaching and Research Hospital, Department of Obstetrics and Gynecology, Istanbul, Turkey \\ ${ }^{3}$ Department of Pathology, Istanbul Faculty of Medicine, Istanbul University, Istanbul, Turkey \\ Email: *alged_1971@yahoo.com
}

Received 27 May 2013; revised 30 June 2013; accepted 8 July 2013

Copyright (C) 2013 Volkan Ulker et al. This is an open access article distributed under the Creative Commons Attribution License, which permits unrestricted use, distribution, and reproduction in any medium, provided the original work is properly cited.

\begin{abstract}
Placental site trophoblastic tumor is a rare sub-group of gestational trophoblastic neoplasia. There is a wide clinical spectrum of presentation and behaviour ranging from a benign condition to an aggressive disease with a fatal outcome. We report a case of placental site trophoblastic tumor in 23-year-old women with irregular vaginal bleeding during postpartum lactation period. In addition to persistent low level B-hCG titers, ultrasound examination revealed a suspicious low-echoic area in the myometrium consistent with gestational trophoblastic disease. After histopathological examination of the specimen achieved by ultrasound-guided dilatation and curettage of the uterus, the placental site trophoblastic tumor diagnosis was made and subsequently total abdominal hysterectomy was performed. The patient had an uneventful recovery, and no recurrence was detected for $\mathbf{4 0}$ months in the follow-up period.
\end{abstract}

Keywords: Gestational Trophoblastic Disease; Placental Site Trophoblastic Tumor; Hysterectomy

\section{INTRODUCTION}

Placental-site trophoblastic tumour (PSTT) is the rarest form of gestational trophoblastic neoplasms, accounting for only $0.5 \%$ of patients with gestational trophoblastic disease worldwide [1]. This tumour develops from intermediate trophoblast, unlike choriocarcinoma, which arises from villous trophoblast. PSTT might follow any type of pregnancy event and not infrequently becoming clinically apparent even years later [2]. The tumor be-

\footnotetext{
"Corresponding author.
}

haviour can vary as confined benign lesion to the uterus to systemic metastasis in an aggressive malignant mode. Localized PSTT's clinical signs are irregular vaginal bleeding, amenorrhea and uterine enlargement [3]. According to a study of 34 patients, lungs are the most common site of metastases. In addition, the vagina, lymph nodes, brain, liver, ovaries, pancreas, and bladder are other sides complicated with metastases [4]. PSTT is usually resistant to chemotherapy and surgery is required in most cases. Hysterectomy is the choice of treatment for local disease confined to uterus. Patients with multiple distant metastases need chemotherapy; however, the disease may progress despite treatment with aggressive multi-agent chemotherapy [5].

\section{CASE PRESENTATION}

A 23-year-old woman gravida 2, para 1, abortion 1 presented to her primary care physician with complaint of irregular vaginal bleeding at lactation period in which beginning after 16 months of caesarean delivery. Two months later, because of persistent clinical complaints and low-level B-hCG titers in the range of 65 - $95 \mathrm{IU} / \mathrm{L}$, the patient was referred to our gynecologic oncology clinic. On admission her B-hCG concentration was 86 IU/L. Endovaginal sonography demonstrated a $2 \mathrm{~cm}$ diameter hypoechoic mass in the anterior wall of the uterus which was well-circumscribed and $2 \mathrm{~mm}$ close to the serosal surface (Figure 1(A)). A diagnosis of PSTT was established upon histological examination of the tissue sample obtained by dilatation and curettage of the uterus. Computed tomography of the chest and magnetic resonance imaging (MRI) of upper and lower abdomen did not show any evidence of distant spread. T1- and T2weighted MR images demonstrated a 2-cm-diameter 
mass in the anterior wall of the uterus adjacent to the endometrium (Figure 1(B)). Following investigations, she was treated surgically with total abdominal hysterictomy and bilateral pelvic lymph node dissection. On cut sections, a dark red mass was seen mainly in the anterior wall of the myometrium (Figure 1(C)). The histopathological evaluation of the tumor demonstrated fragments of endometrium and myometrium with infiltration of the myometrium by sheets of mitotically active intermediate trophoplast cells with eosinophilic cytoplasm and ovoid nuclei which is characteristic of PSTT (Figure 1(D)). In addition to the mentioned histological characteristics above, the tumor cells were strongly positive for human placental lactogen (hPL). The hCG-reaction was also positive, but less intensely than was hPL. Ki-67 expression was weak and present in only $15 \%$ of cells. After surgery, her B-hCG concentration normalised without any further intervention. The patient had no symptoms in the postoperative period and she has had no symptoms for 40 months.

\section{DISCUSSION}

Placental site trophoblastic tumours might follow any type of pregnancy event, not infrequently becoming clinically apparent even after years, and there is great

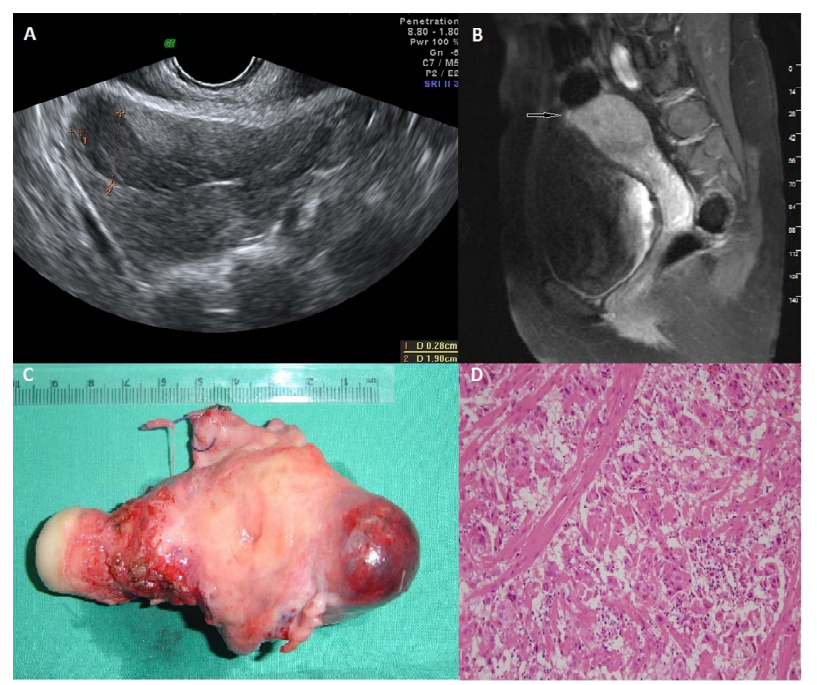

Figure 1. (A) Endovaginal sonography demonstrated a $2.5 \mathrm{~cm}$ diameter hypoechoic mass in the anterior wall of the uterus. (B) Coronal T1-weighted fat suppressed MR image shows 2.5-cm mass (small arrows) in superior uterine fundus adjacent to endometrium. Most of mass is slightly hyperintense to outer myometrium. (C) Macroscopic features of placental site trophoblastic tumour: a dark red mass was seen mainly in the anterior wall of the myometrium. (D) Low-power view of placental site trophoblastic tumour (H\&E, X100). The predominant type of cell in PSTT is implantation-site-intermediate trophoblast which is characteristically a large polyhedral cell with irregular hyperchromatic nuclei. The neoplastic cells extensively infiltrate the myometrium. variability in its malignant aggressiveness. The majority of the 200 cases published in the English literature so far have had good prognosis but rapidly metastasizing, and fatal cases have also been reported. In a review of 62 PSTT women treated at three gestational trophoblastic disease centres in United Kingdom, 23\% patients died resulting in a probability of overall survival of $73 \%$ at 5 years and $70 \%$ at 10 years [6]. Previous reports have demonstrated that age older than 35 years, deep myometrial invasion $(>1 / 2)$, high mitotic rate $(>5$ per 10 HPF), clear cytoplasm, coagulative necrosis, vascular space involvement, prior term pregnancy, and increased interval from previous pregnancy are important factors in the prognosis of these tumors [7]. However, Schmid et al. reported that only time ( $>48$ months) since antecedent pregnancy remained significantly associated with multivariate analyses after adjustment for the FIGO score [6].

In our case, younger age, stage 1 disease, shorter time interval (16 months) from the preceding pregnancy, low B-hCG levels ( $<100 \mathrm{IU} / \mathrm{L})$, low mitotic rate, and negative lymph nodes were good prognostic factors. However, deep myometrial invasion, and the previous term pregnancy were associated with poor prognosis.

The first symptoms of the disease are usually seen after delivery or abortion. As described in the literature and particularly seen in our case, the most common symptom is vaginal bleeding [8]. Other presentations include amenorrhea, abdominal pain, and postmenopausal bleeding [9]. Unlike choriocarcinoma, the level of serum B-hCG in PSTT correlates neither with tumor burden, nor with the malignant behaviour. Thus, B-hCG appears to have no predictive value and disease may still progress even if levels are not raised. The range of serum B-hCG concentration at diagnosis in $69 \%$ is below $1000 \mathrm{IU} / \mathrm{L}$ [6]. The explanation of this moderated elevation of B-hCG is that these cells produce primarily more hPL and less B-hCG.

PSTT is staged according to the FIGO staging of gestational trophoblastic disease. On the other hand, the behaviour of PSTT has not been well predicted by the FIGO 2000 scoring system for gestational trophoblastic disease and needs to be considered separately [10]. Although, some publications reported a spontaneously regress after curettage, the standard treatment of local PSTT is total abdominal hysterectomy with lymph node dissection. Adnexal micrometastases are rare (3\%) and the protection of patients' ovaries is recommended in women particular under 40 years of age [5]. Conservative surgical treatment may have a role in patients with stage I tumors who wish to conserve fertility, as long as their tumors do not have unfavourable prognostic features. This usually consists of local excision by hysterotomy with close follow-up with serial serum B-hCG levels, pelvic examination, and radiologic imaging such as MRI [11]. In cases of conservative surgery, secondary 
operation may be necessary because the resection margins may be involved and pathologic findings may demonstrate the presence of potentially poor prognostic factors, or multifocal disease may be revealed [12].

The role of adjuvant chemotherapy and the optimal treatment of metastatic disease remain uncertain. In patients with low stage disease, chemotherapy is generally not necessary and cannot be substituted for surgical treatment of the primary, particularly since treatment with chemotherapy, as used for other gestational trophoblastic tumors, is usually ineffective in PSTTs. Although most patients with placental-site trophoblastic tumours can be cured, a substantial number of patients have recurrent disease. For such patients, Schmid et al. reported that the outcome is poor with only 33\% achieving long-term remission [6]. In this series, patients with stage I disease had a 10 -year probability of overall survival of $90 \%$ and did not benefit from postoperative chemotherapy. By contrast, patients with stage II, III, and IV disease required combined treatment with surgery and chemotherapy; probability of overall survival at 10 years was 52\% for patients with stage II disease and $49 \%$ for stage III or IV disease.

Very few data are currently available on the genetics of PSTT. Although these tumours are less often associated with an antecedent molar pregnancy, it has been confirmed by using DNA analysis that, like choriocarcinoma, they may arise from both HM (hydatidiform mode) and normal term pregnancy [8]. Karyotype of PSTT is diploid, but few cases of tetraploidy have been reported [13]. Genetic analysis showed an XX genomic composition in $89 \%$ of PSTT. Most of PSTT were derived from the antecedent female conceptus and were likely to have possessed a functional paternal X chromosome.

In the study of methylation status analysis at the human androgen receptor (AR) gene locus in the PSTT in which the paternal $X$ chromosome was identifiable, the paternal AR locus was hypomethylated while the corresponding maternal locus was hypermethylated [14].

In conclusion, PSTT is potentially highly curable if disease is confined to uterus and treated appropriately. Immediate hysterectomy is required for patients without metastasis until reliable prognostic indicators for PSTT are clearly understood.

\section{REFERENCES}

[1] Kohorn, E.I. (2009) World-wide results of therapy for gestational trophoblastic disease. Gynecologic Oncology, 112, 85.

[2] Scully, R.E. and Young, R.H. (1981) Trophoblastic pseudotumor: A reappraisal. American Journal of Surgical Pathology, 5, 75-76. doi:10.1097/00000478-198101000-00010

[3] Kim, S.J. (2003) Placental site trophoblastic tumour. Best Practice \& Research Clinical Obstetrics \& Gynaecology, 17, 969-984. doi:10.1016/S1521-6934(03)00095-6

[4] Papadopoulos, A.J., Foskett, M., Seckl, M.J., McNeish, I., Paradinas, F.J., Rees, H. and Newlands, E.S. (2002) Twenty-five years' clinical experience with placental site trophoblastic tumors. The Journal of Reproductive Medicine, 47, 460-464.

[5] Chang, Y.L., Chang, T.C., Hsueh, S., Huang, K.G., Wang, P.N., Liu, H.P. and Soong, Y.K. (1999) Prognostic factors and treatment for placental site trophoblastic tumorreport of 3 cases and analysis of 88 cases. Gynecologic Oncology, 73, 216-222. doi:10.1006/gyno.1999.5344

[6] Schmid, P., Nagai, Y., Agarwal, R., Hancock, B., Savage, P.M., Sebire, N.J., Lindsay, I., Wells, M., Fisher, R.A., Short, D., Newlands, E.S., Wischnewsky, M.B. and Seckl, M.J. (2009) Prognostic markers and long-term outcome of placental-site trophoblastic tumours: A retrospective observational study. The Lancet, 374, 48-55. doi:10.1016/S0140-6736(09)60618-8

[7] Baergen, R.N., Rutgers, J.L., Young, R.H., Osann, K. and Scully, R.E. (2006) Placental site trophoblastic tumor: A study of 55 cases and review of the literature emphasizing factors of prognostic significance. Gynecologic Oncology, 100, 511-520.

[8] Palmieri, C., Fisher, R.A., Sebire, N.J., Smith, J.R. and Newlands, E.S. (2005) Placental-site trophoblastic tumour: An unusual presentation with bilateral ovarian involvement. The Lancet Oncology, 6, 59-61.

[9] Feltmate, C.M., Genest, D.R., Wise, L., Bernstein, M.R., Goldstein, D.P. and Berkowitz, R.S. (2001) Placental site trophoblastic tumor: A 17-year experience at the New England Trophoblastic Disease Centre. Gynecologic Oncology, 82, 415-419. doi:10.1006/gyno.2001.6265

[10] International Federation of Obstetrics and Gynecology Oncology Committee (2002) FIGO staging for gestational trophoblastic neoplasia. International Journal of Gynecology \& Obstetrics, 77, 285-287.

[11] Leiserowitz, G.S. and Webb, M.J. (1996) Treatment of placental site trophoblastic tumor with hysterotomy and uterine reconstruction. Obstetrics \& Gynecology, 88, 696699. doi:10.1016/0029-7844(96)00124-X

[12] Pfeffer, P.E., Sebire, N., Lindsay, I., McIndoe, A., Lim, A. and Seckl, M.J. (2007) Fertility-sparing partial hysterictomy for placental-site trophoblastic tumour. The Lancet Oncology, 8, 744-746. doi:10.1016/S1470-2045(07)70243-7

[13] Xue, W.C., Guan, X.Y., Ngan, H.Y., Shen, D.H., Khoo, U.S. and Cheung, A.N. (2002) Malignant placental site trophoblastic tumor, a cytogenetic study using comparative genomic hybridization and chromosome in situ hybridization. Cancer, 94, 2288-2294. doi:10.1002/cncr.10424

[14] Usubütün, A. (2011) Gestational trophoblastic disease. Turkiye Klinikleri Journal of Medical Oncology Special Topics, 4, 48-53. 